$B B$
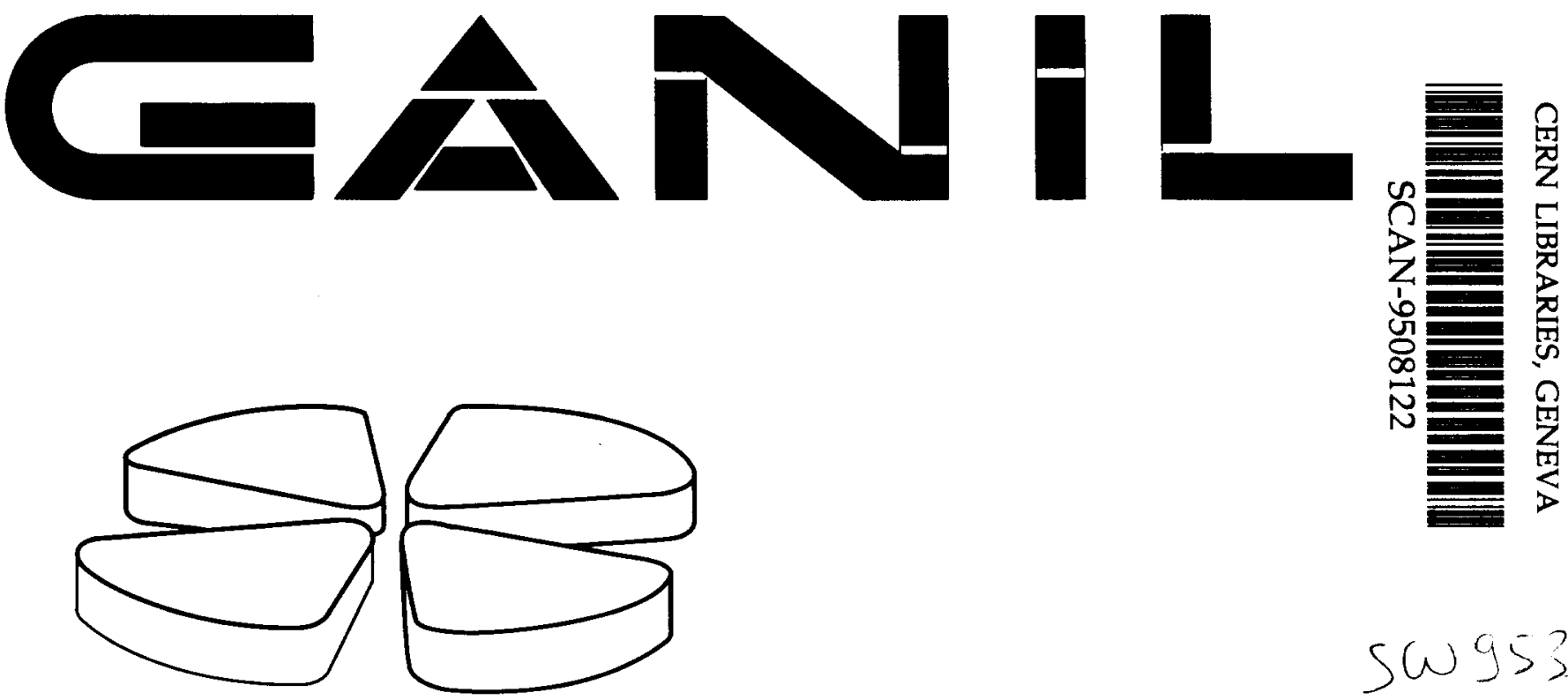

569533

\title{
Regularity and chaos in Vlasov evolution of Nuclear Matter
}

B. Jacquot ${ }^{1}$, A. Guarnera ${ }^{1,2}$, Ph. Chomaz ${ }^{1}$, M. Colonna ${ }^{1,2}$

1) GANIL, B.P. 5027, F-14021 Caen Cedex, France

2) Laboratorio Nazionale del Sud, Viale Andrea Doria, I-95129 Catania, Italy

Submitted to Physical Review C 


\title{
Regularity and chaos in Vlasov evolution of Nuclear Matter
}

\author{
B. Jacquot ${ }^{1}$, A. Guarnera ${ }^{1,2}$, Ph. Chomaz ${ }^{1}$, M. Colonna ${ }^{1,2}$ \\ 1) GANIL, B.P. 5027, F-14021 Caen Cedex, France \\ 2) Laboratorio Nazionale del Sud, Viale Andrea Doria, I-95129 Catania, Italy
}

July 13,1995

\begin{abstract}
In this article we perform a careful analysis of the mean-field dynamics inside the spinodal instability region. As a main finding, we show that, conversely to some recently published results, the mean-field evolution appears mostly regular over a long time scale, while some disorder is observed only very late, when fragments are already formed. This onset of chaos can be related to the fragment interaction which induces some coalescence effects. Even at the latest considered stage, chaos does not appear as fully developed since a strong hierarchy of the different unstable modes still remains, in particular preserving small wave lengths to develop. Moreover, we show that these results and in particular the time scale over which the chaos start to develop are very sensitive to the range of the considered force.

All the presented results support the various analyses of spinodal instabilities obtained using stochastic mean field approaches.
\end{abstract}

PACS numbers: 25.70.Pq, 24.60.Lz, 21.65. $+\mathrm{f}$ 


\section{Introduction}

Mean field equations are known to be highly non linear, because of the selfconsistency in the calculation of the potential. Therefore they are expected to exhibit all the phenomenology of non-linear processes. For example, a nucleus traveling at constant velocity can be seen as a solitary wave (or soliton) solution of the mean-field equations. The existence of strong non-linearities makes the mean-field dynamics a typical candidate to exhibit a chaotic behaviour. However it should be noticed that this is actually a very delicate point. For example, we know that even at finite temperature, the mean field dynamics can present regular collective motions, such as the giant resonances, which have been predicted theoretically and observed experimentally.

Recently, the investigations about the collective properties of the mean-field dynamics have been extended to consider unstable situations, inside the spinodal region of the nuclear matter phase diagram $[1,2,3]$. The studies of ref. $[4,5$, 6] have shown that the spinodal decomposition, simulated through full meanfield calculations, is strongly influenced by the existence of very unstable modes, which are equivalent to zero-sound waves. On the other hand opposite results advocating a very fast $(t<20 \mathrm{fm} / \mathrm{c}$ ) dominance of chaos in unstable systems have been reported $[7,8]$. Therefore it is an important issue to clarify whether or not the dynamics of a system inside the spinodal region is fully chaotic. Indeed, if the conclusions of ref.s $[7,8]$ are valid, all the results on the possibility to preserve some memory of the initial instabilities in the final fragmentation pattern $[4,5,6]$ could not be correct. We will show in this article that this is not the case; in fact a careful analysis of the very same calculations as the one published in ref $[7,8]$ leads to the conclusion that the dynamics of an unstable system presents some strong regularity during the whole fragmentation stage while some partial disorder is then produced mostly because of the random coalescence of the formed fragments.

Indeed, in a recent comment [9] it has been shown that some of the results, analyses and conclusions presented in ref.s $[7,8]$ were affected by a numerical error; actually the corrected results show a much more regular pattern, understandable in terms of linear instabilities, during the early evolution of the system. Therefore, a detailed analysis of when and how the chaos appears is still missing.

In this article we will show that the most important unstable degrees of freedom follow the regular behaviour of decoupled unstable oscillators until large times, whereas less important degrees of freedom present some disorder after slightly shorter times. We will demonstrate that this conclusion is valid for the dynamics of a pure normal mode of the linear response approach and also for any mixing, such as a randomly initialized perturbation.

As far as the sensitivity to the initial conditions is concerned, we will point out that, in presence of instabilities, the exponential growth of small differences is not specific of a chaotic regime, since it can be obtained also in a regular regime of decoupled unstable oscillators. Indeed we will show that the divergence of two different trajectories is simply due to the amplification of normal modes and 
not to the strong occurrence of chaos. On the same line, we will show that the Lyapunov exponents found in ref.[7], or extracted from the divergence of different trajectories initialized at random, are nothing but the growth time of the most unstable modes and can also be understood with a simple model of decoupled unstable oscillators. This conclusion also holds as far as the variation of these exponents with density and temperature is concerned. These findings confirm the strong influence of zero-sound waves also in unstable mean-field dynamics; therefore it is not worth relating the values obtained for the Lyapunov exponents to some universal behaviour of bifurcating or chaotic systems, as it is sometimes done.

In such a context, we will show that the recognition of the normal modes of the system is an essential tool to disentangle between a regular and a disordered regime. Introducing a novel way to analyze the regularity or the presence of chaos in an unstable system we will be able to clearly separate a regular regime during the fragmentation of the system and a (less-regular) chaotic one, after a long time. We will relate this onset of chaos to the occurrence of coalescence effects between the already formed fragments. In particular we will insist on the fact that small wave lengths do not present strong fluctuations because they are not unstable and are not affected by the disorder generated by the coalescence mechanism.

As far as fragment size distributions are concerned, we will stress that definite conclusions cannot be reached considering closed systems, such as in ref.[8], because of the strong dependence of the results upon the arbitrary time considered for the analysis, and upon the threshold density used in the definition of a cluster. Reliable results can be obtained only considering either expanding systems or finite geometries $[10,11]$. Finally, we will stress the importance of the range of the nuclear force in fragmentation by spinodal decomposition and we will show how the conclusions on the presence of chaos depend on it. In particular, we will discuss how the different time and size scales are affected by this parameter.

\section{Analysis in terms of normal modes}

Let us consider the lattice simulations developed in ref. [12] and used in ref.s $[4,7,8]$, i.e. the case of two-dimensional nuclear matter evolving under the action of a Skyrme-like density dependent mean-field potential: $U=A \rho+B \rho^{\sigma}$. In order to take into account the finite range of the nuclear interaction, this potential has been folded with a gaussian-like function. The half width at half maximum of this function was chosen to be $a \approx 0.3 \mathrm{fm}$ in ref.s[7,8], while, to mimic the 3D nuclear quantum case, in ref.[4] we use a longer range, $a=0.9$ $\mathrm{fm}$. We will quote these two interactions as $S$ (for short range) and $L$ (for long) respectively (see Fig.1). Moreover the potential is averaged along the $\mathrm{y}$ direction, so that waves can only propagate, or be amplified, along the $\mathrm{x}$-axis.

For a piece of infinite nuclear matter (periodic boundary conditions are applied to the box used in the calculations) the normal modes are plane waves charac- 
terized by a wave number $k$. Their dispersion relations, in the linear response limit of the semi-classical mean-field evolution, are shown in Figure 1 , for both interactions, for a given point inside the spinodal instability region of the phase diagram $\left(\rho=\rho_{0} / 2, T=3 \mathrm{MeV}\right)$. As discussed in ref.s [1,2], an important feature of the dispersion relation is the presence of an ultra-violet cut-off induced by the finite range of the nuclear interaction. It should be noticed that the long range interaction $\mathrm{L}$ has been constructed in such a way that the associated dispersion relation is very close to the realistic one obtained in ref. [13].

As far as the mean-field dynamics in unstable situations is concerned, refs. [7, 8] are reporting very strong sensitivity to the initial conditions for the propagation of the normal modes. However, it has been shown in a recent comment that this was due to an error in the initialization procedure [9] which was artificially exciting, from the very beginning, small wave length modes. Since these modes are very unstable when a short range interaction is used, they soon dominate the dynamics leading to the observations reported in ref.s $[7,8]$. The correct evolutions of some normal modes are reported in Fig. 2 for two slightly different initial densities and for the two considered interactions. As discussed in the comment [9], the corrected dynamics of a single RPA eigenmode appears very regular, as it can be also seen from the Fourier analysis displayed in Fig.3. We will come back to these points in the following chapter because the observed regularity of the corrected dynamics of the normal modes strongly modifies also the analysis about the Lyapunov exponents and the conclusions reported in ref.s $[7,8]$.

In order to study completely the characteristic of the considered non-linear dynamics it is not sufficient to look at the propagation of normal modes, because these are very peculiar initial conditions. Therefore we have studied the dynamics of randomly initialized trajectories. The average density was chosen to be equal to $0.4 \rho_{0}$. We will see that the same conclusions about the occurrence of regular motion hold during a rather long time.

If one looks at the evolution of two randomly initialized systems, they appear very different, expecially in the case of the short range interaction, (see Fig.4) and one may be tempted to speak about a chaotic behaviour. However, this is a delicate point, since even in the case of independent unstable normal modes, one would observe the same features on the r-spectrum (see Figure 5). Therefore, in order to carefully investigate about the presence of chaos, we have performed a normal mode analysis, i.e. an analysis in terms of the Fourier transform $\sigma_{k}$ of the density fluctuations.

A first approach to pin down the occurrence of a regular evolution or of a chaotic dynamics is to try to define some equivalent of a phase space or a Poincaré plot. In the present case, for a given mode $k$, we can associate to each trajectory, at each time, a couple of values $\left(\sigma_{k}, \dot{\sigma}_{k}\right)$; then, on the same plot, we

can show for many different events the various points $\left(\sigma_{k}^{(n)}, \dot{\sigma}_{k}^{(n)}\right)$ taken every 5 $\mathrm{fm} / \mathrm{c}$, from the initial time until $\mathrm{t}=50 \mathrm{fm} / \mathrm{c}$ for the $S$ force and $200 \mathrm{fm} / \mathrm{c}$ for the $\mathrm{L}$ force. These plots are shown on Fig.6, for both forces (S and L) and various 
$k$. This way to analyze the results displays a regular pattern. Indeed, for all the considered unstable modes $\dot{\sigma}_{k}^{(n)}$ seems to be a unique function of $\sigma_{k}^{(n)}$. These plots emphasize the linearity of the initial evolution, since one simply observes a straight line.

Another way to analyze in more detail a complex unstable evolution is to look at the relation between the initial amplitude in a considered degree of freedom and some final values. Here we can study this correlation as a function of different final times. To construct these plots we have considered random initial conditions with a white noise and we have run 200 events (each of them giving a point in the diagram). Figures 7-8 display, for different modes (around the most unstable one), the relation between the initial and final (at time $t=50 \mathrm{fm} / \mathrm{c}$ for the short range force and $t=100 \mathrm{fm} / \mathrm{c}$ for the long range interaction) modulus of the Fourier transform $\sigma_{k}$. The phases are also plotted in Fig.s 7-8. For the most unstable mode the modulus $\sigma_{k}$ is shown for different final times, in Figure 9. From these figures it is clear that the unstable modes are following a very regular behaviour up to long time since the initial and final amplitudes $\sigma_{k}$ appear strongly correlated. Moreover, the fact that the correlation takes the form of a simple straight line indicates that the dynamics is mostly linear. After this rather long regular regime (during $70 \mathrm{fm} / \mathrm{c}$ and more than $200 \mathrm{fm} / \mathrm{c}$ for the forces $S$ and $L$ respectively) some disorder sets in and the correlation starts to present some fluctuations. We can notice that the appearance of some disorder coincides with the beginning of the saturation of the amplitudes associated with the most unstable modes. This saturation marks the end of the fragment formation (when most of the local various densities are out of the spinodal region) (see Fig.4) and the beginning of the coalescence of the formed fragments. This demonstrates that the most unstable collective modes are very robust and that their dynamics is strongly decoupled. It is also clear that the modes which are less unstable (or even not unstable) start to present some disorder earlier than the most unstable ones. It can be seen that some disorder sets in when the amplitude of the considered mode is much smaller than the one of the most unstable ones. Indeed, at this point the linear response treatment in the particular considered channel starts to loose its validity.

The obtained results recall the survival of collective motion at high temperature which has been observed for stable situations, as in the case of the hot Giant Dipole Resonance. This is a novel illustration of the fact that fully regular and fully chaotic regimes are only two extremes and that in general we must expect to observe an intermediate situation, the weaker modes becoming chaotic sooner than the more robust ones. In the case of spinodal decomposition, since the most robust modes are the most unstable ones, they rapidly dominate and therefore the dynamics keeps over a long time scale the memory of a mostly deterministic behaviour. This is stressed by the comparison of the complete mean-field dynamics with a simple calculation using decoupled unstable oscillator, as shown in Fig.5.

It is also important to notice that even for very large times, when some disorder is present, one can still observe that the dynamics is not yet fully chaotic, in 
the sense that a strong hierarchy between the various modes is kept as a memory of the instabilities which were developing during the early stage. This is shown in Fig.s 10-11-12-13, where the relation between the initial and the final fluctuation $\sigma_{k}$ is reported for different times and different wave numbers (left part). One can see again that, while during the early evolution the dynamics is clearly regular for all the modes, the most unstable one remains dominant when the other modes start to present some disorder and the amplitude of the various modes directly reflects the relative importance of the various imaginary RPA frequencies. Then when the most unstable modes saturate because the fragments are formed, they start to be coupled with lower $k$ values, because of the coalescence mechanism which reduces the fragment number. It is important to notice that the high frequencies are not affected by this process and indeed their associated amplitude remains small and therefore small clusters are not formed by spinodal instabilities. This is demonstrated also in Fig.s 10-11-12-13 (right part), which shows the distribution of $\sigma_{k}$ for various $k$ as a function of time.

In references $[4,6]$ the very same phenomenology has been reported. Indeed, the authors were looking at the $\sigma_{k}$ averaged over many events in the case of mean-field evolution, with and without additional stochasticity, and the same features have been observed: the correlation function $\left\langle\sigma_{k}\right\rangle$ presents a strong peak even at very long times. The position of the peak seems directly related to the most unstable modes of the linear response and shifts only slowly towards lower $k$ values because of coalescence effects. Moreover, it should be noticed that the same results have been reported in the case of an exact simulation of a classical fluid inside the spinodal zone [14]. Indeed it has been shown that the domain formation in a classical liquid-gas phase transition occurs according to the most unstable zero-sound waves. Therefore the above discussed features are not an artefact of the mean field approximation but are rather a general property. Moreover, they are well-known in the case of classical fluids such as binary alloys, for which they have been even observed experimentally [15].

\section{Lyapunov exponents}

Having in mind the above observations, one may wonder about the meaning of the Lyapunov exponent reported in ref.[7,8]. Indeed defining a distance between trajectories as the modulus of the difference of densities in r-space:

$$
d(t)=\sum_{i}\left|\rho^{(1)}\left(x_{i}, t\right)-\rho^{(2)}\left(x_{i}, t\right)\right| / N_{c}
$$

where $N_{c}$ is the number of cells along the $x$ axis, it is possible to define a timedependent Lyapunov exponent [7]:

$$
\lambda(t)=\frac{\ln [d(t) / d(0)]}{t} .
$$


Let us consider two trajectories with slightly different densities and let us study the evolution of a single RPA mode. In ref.[8], looking at the $\lambda(t)$ curve, the authors show the existence of a plateau, which has been interpreted as a signature of a chaotic regime. As we discussed already, because of an error in the initialization procedure (see ref.[9]) used in ref.[7], the most unstable modes were always excited in the initial conditions, together with the considered mode. Actually, if one compares the exponents reported in ref.s $[7,8]$ with the prediction of the imaginary frequency of the most unstable modes obtained in the linear response approximation (see Fig.1 ), we can see that the Lyapunov exponent of ref.s $[7,8]$ is nothing but the inverse of the characteristic growing time of the most unstable mode. It should be noticed that also the density and the temperature dependence found in ref.s $[7,8]$ corresponds to the characteristic behaviour of the most unstable mode, predicted by the linear response theory (see Fig.14).

The conclusion reported in ref.[7] about the independence of the Lyapunov exponent on the wave number $k$ therefore appears as an artefact of the initialization procedure which was always exciting the most unstable modes. The Lyapunov exponents extracted from the corrected dynamics of a single eigenmode are either zero, in the present case, or equal to the amplification rate of the considered mode, predicted by the linear response approach (if the initial trajectories differ also by the amplitude of the mode).

To get a deeper insight into the meaning of the Lyapunov exponents one can consider the dynamical evolution of two randomly initialized trajectories. This is shown in Figure 15. One can observe a plateau showing the occurrence of an exponential growth of differences between the trajectories, but again the value of the Lyapunov exponent appears to be closely related to the largest imaginary frequency of the linear response.

This is clearly demonstrated in Fig.15, which also presents the result obtained in the case of decoupled unstable oscillators (dashed line) and which shows the same characteristic Lyapunov exponent as the complete calculation (full line).

Moreover we can see that the end of the plateau of $\lambda(t)$, defined as the fragmentation time $\tau_{\text {frag }}$ in ref. [8], does not correspond to a chaotic regime, as one can see from Fig.s 7-13, while some chaos slowly gets in afterwards. Indeed, it can be seen that the end of the plateau is associated with the beginning of the saturation of the most unstable modes and still corresponds to a very regular regime. This conclusion is strengthen by a simple calculation using decoupled oscillators (i.e. a regular dynamics) but taking into account also the non-linearity coming from the saturation. Indeed this calculation is able to perfectly fit the rise and fall of the Lyapunov exponent.

Therefore one can see that, by performing a detailed analysis in terms of normal modes, one gets to conclusions which are very much in line with the discussions of ref. [5] and which are orthogonal to the finding of ref. [7, 8]. Indeed we have demonstrated that strong regularities survive until large times. The same behaviour has been observed in the complete many-body simulations of the spinodal decomposition of a classical gas reported in ref. [14]. 


\section{Fragment size distribution}

Let us then investigate the fragment size distribution. When considering a closed geometry, the fragments cannot be defined without ambiguities, because one needs to introduce a threshold density $\rho_{c u t}$ above which a given region is considered occupied. Moreover, one needs to choose a given time at which the analysis is performed. These are the reasons why many efforts have been devoted to consider expanding infinite nuclear matter or finite systems (see ref. $[5,10,11,16]$ ). Indeed in such cases after some time, usually called the freeze-out time, the fragments are well separated and the fragment size distribution can be univocally defined. However, ref. [8] is presenting fragment size distributions for the considered infinite system after a time very close to $\tau_{\text {frag }}$. Considering $\frac{\rho_{c u t}}{\rho_{0}}$ around 0.05 or 0.1 , distributions that could be akin to power laws or exponential laws are obtained in ref.[8] and strong statements about the relevance of these distributions in connection with experimental findings, statistical models, phase transitions and critical phenomena are made. However these conclusions strongly differ from the ones obtained in fully dynamical calculations either in expanding geometries or in finite systems $[5,10,11,16]$. The presence of chaos was the interpretation of the broad distribution observed in ref [8], but, on the basis of the previous analysis demonstrating the persistence of regular motion, this argument does not hold anymore.

In fact, it can be easily seen that this observation is a consequence of the adopted definition for the fragments. Indeed in Fig. 16 we show that the results change drastically with $\rho_{\text {cut }}$. We can see that, depending on this parameter, we can obtain almost any shape for the fragment distributions, from the dominance of small clusters, when $\rho_{\text {cut }}$ is high, to the presence of only one large fragment. The parameter $\rho_{c u t}$ acts almost as a site occupancy parameter in a site percolation calculation. Moreover, going to a later time the fragment size distribution gets completely different. Therefore the observed size distribution cannot be defined in a reliable way in the considered closed geometry and does not contain strong connections with chaos or phase transitions.

Fragment size distributions obtained in open systems are discussed in ref.s $[10$, 11]. They are shown to present a large mass tail coming from mode beating and from final state interactions (coalescence effects), but also display a small size cut-off due to the fact that large $k$ are not unstable and are not populated during the disordered phase associated with coalescence effects (see Fig.1). The same feature has been observed in molecular dynamics simulations of a classical liquidgas phase transition [14], showing again that this is not an artefact of one-body approaches.

\section{Conclusions}

In the present article we have shown that all the arguments presented in ref. $[7,8]$ in favour of a chaotic creation of fragments do not remain after the correction 
of some results and a critical analysis of the involved dynamics. Conversely the presented investigation gives more credit to the results obtained in transport theories, showing some reminiscence of the linear instability stage in the final partition of the system. Moreover, we have stressed the role played by the range of the nuclear interaction in the fragmentation pattern; this appears to be a crucial point as far as the qualitative and quantitative discussions of spinodal decomposition are considered [5]. Indeed investigating realistic interactions, one gets to the conclusion that the most unstable wave length in the spinodal region is around $10 \mathrm{fm}$, associated with a characteristic growing time $\tau=30-40 \mathrm{fm} / \mathrm{c}$, while the short range interaction of ref. [8] predicts lengths and time scales 3 times smaller. For example, the numbers quoted in Table 1 for the instability time, for the long range interaction, are not in contradiction with the use of mean field approaches, since (for densities larger than $0.2 \rho_{0}$ ) this time is longer than the single particle time $\tau_{s p}$ associated with the Fermi velocity. Moreover our conclusions are confirmed by exact simulations of classical systems undergoing liquid-gas phase transitions, far from the critical point [14].

We have demonstrated that, under the action of a force with a long range attractive part, the first stage of the fragment formation in spinodal decomposition is not dominated by chaos, so that the phase space cannot be assumed to be populated in a statistical manner. This is confirming some previous analyses, using a full dynamical calculation of the stochastic mean-field type, which show a strongly structured correlation function $[5,11]$. In the present analysis we show that some disorder starts to play a role when the already formed fragments start to interact. However it should be recalled that this occurs over a very long time [16], showing that the statistical equilibration of the fragment partition is a very slow process. Indeed even at the latest times considered here the fluctuations in the different modes still exhibit the hierarchy introduced by the regular amplification of instabilities. Moreover, it was already shown in ref.s $[5,10,11,16]$ that considering finite size expanding systems introduces a time scale, i.e. a freeze-out time, which prevents the system to reach this equilibrium. These are known facts in the physics of spinodal decomposition, for instance in the case of the growth of domains in bynary alloy systems, for which a direct chaotic feeding of the whole accessible phase space does not correspond to the experimental and theoretical findings $[15]$.

Acknowledgements: We would like to thank M.Baldo, G.F.Burgio and A.Rapisarda for numerous and very useful discussions.

This work is supported in part by the Commission of the European Community, under Contract No. ERBCHBI-CT-930619. 


\section{References}

[1] H. Heiselberg, C.J. Pethick, and D.G. Ravenhall, Ann. Phys. 223 (1993) 37

[2] M.Colonna, Ph.Chomaz, J.Randrup, Nucl. Phys. A567 (1994) 637.

[3] Ph. Chomaz and M. Colonna, Phys. Rev. C49 (1994) 1908

[4] M. Colonna, G.F. Burgio, Ph. Chomaz, M. Di Toro, and J. Randrup, Phys. Rev. C47 (1993) 1395

[5] Ph.Chomaz, M.Colonna, A.Guarnera and B.Jacquot, Nucl. Phys. A583 (1995) 305; M.Colonna, Ph. Chomaz and A.Guarnera, submitted to Nucl. Phys. A.

[6] G.F.Burgio, Ph.Chomaz, M.Colonna and J.Randrup, Nucl. Phys. A581 (1995) 356.

[7] G.F. Burgio, M. Baldo and A. Rapisarda, Phys. Lett. B321 (1994) 307.

[8] M. Baldo, G.F. Burgio and A. Rapisarda, Phys. Rev. C51 (1995) 198.

[9] B.Jacquot, M.Colonna, Ph.Chomaz, A.Guarnera, GANIL preprint P95 15, submitted to Phys. Lett. B

[10] M.Colonna, Ph.Chomaz, A.Guarnera and B.Jacquot, Phys. Rev. C51 (1995) 2671.

[11] A.Guarnera, M.Colonna and Ph.Chomaz, GANIL preprint P95 13, submitted to Phys. Rev. Lett.

[12] Ph. Chomaz, G.F. Burgio, and J. Randrup, Phys. Lett. B254 (1991) 340; G.F. Burgio, Ph. Chomaz, and J. Randrup, Nucl. Phys. A529 (1991) 157

[13] S.Ayik, M.Colonna, Ph.Chomaz, Phys. Lett, B, in press.

[14] B.Jacquot, A.Guarnera, Ph.Chomaz, M.Colonna, GANIL preprint P95 12, submitted to Phys. Rev. Lett.

[15] J.D. Gunton, M. San Miguel and P.S. Sahni, 1983 Phase Transitions and Critical Phenomena vol.8, ed. C. Domb and J.L. Lebowitz (New York: Academic) p. 267

[16] Ph. Chomaz, M. Colonna and A. Guarnera, "Fingerprints of Dynamical Instabilities", proc. of "Int. Workshop on Dynamical Features of Nuclei and Finite Fermi Systems", Sitges (Barcelona), Spain, September 13-17 1993. 


\section{Table Caption}

For zero temperature, the linear response imaginary frequency of the most unstable mode, and the associated growth time $\tau$, are presented as a function of the density, for the $\mathrm{L}$ and the $\mathrm{S}$ interactions. For comparison, the single particle time $\tau_{s p}$ is also reported. 
Figure 1:

Upper part: The dispersion relation for unstable modes, i.e. the imaginary linear response frequencies as a function of the wave numbers, computed at $\rho=\rho_{0} / 2$ and $T=3 \mathrm{MeV}$, in the case of the short range (S) interaction [7].

Solid line: the theoretical predictions obtained analytically (see ref.[2]); points: the actual values extracted from the Vlasov simulation. The folding function $(S)$ is displayed in the insert on the right.

Lower part: The same as before, but with the long range interaction (L) used in ref.[4] to mimic a realistic nuclear force. The dispersion relation is displayed, as a dashed line, also in the upper part, for comparison.

It should be noticed that, in the case of the $\mathrm{L}$ interaction the dispersion relation is very close to the realistic one derived in ref.[13].

Figure 2:

Dynamical evolution of an eigenmode with wave number $k=0.6 \mathrm{fm}^{-1}$, computed for the two forces and to slightly different initial densities, $\rho=0.5 \rho_{0}$ and $\rho=$ $0.51 \rho_{0}$, as a function of time.

Same as Fig.2, but in $k$ space.

Figure 3:

Figure 4:

a) Two dynamical evolutions of a randomly initialized system, computed using the $\mathrm{L}$ force.

b) same as a) for the $\mathrm{S}$ interaction.

Figure 5:

The complete mean field dynamics (left part) is compared with a purely linearized evolution (right part), obtained considering decoupled unstable oscillators, for a randomly initialized system, in the case of the $\mathrm{S}$ force.

Figure 6:

Phase space plot $\left(\sigma_{k}, \dot{\sigma}_{k}\right)$ for different node numbers $n .200$ events are shown. For each event the value of the two variables is plotted every $5 \mathrm{fm} / \mathrm{c}$, from $\mathrm{t}=5$ $\mathrm{fm} / \mathrm{c}$ to $t=50 \mathrm{fm} / \mathrm{c}$ for the $S$ interaction, and from $t=25 \mathrm{fm} / \mathrm{c}$ to $t=200 \mathrm{fm} / \mathrm{c}$ for the $L$ interaction.

Figure 7:

Correlation between the initial and the final values of the magnitude $\sigma_{k}$ and the phase $\Phi$ of the density in $k$ space, computed for different node numbers $n$ around the most unstable one, at time $t=100 \mathrm{fm} / \mathrm{c}$, for the $\mathrm{L}$ interaction. Each point represents an event. 200 events have been considered. 
Figure 8:

Same as Fig.7, for the S interaction, with $t=50 \mathrm{fm} / \mathrm{c}$.

\section{Figure 9:}

Same as Fig.7, for the modulus $\sigma_{k}$, for the most important mode $k=0.6 \mathrm{fm}^{-1}$ $(n=5)$ for the $\mathrm{L}$ interaction (a) and $k=1.7 \mathrm{fm}^{-1}(n=14)$ for the $\mathrm{S}$ interaction (b), presented at different final times.

Figure 10:

Comparison between the amplitudes of the density in $k$ space for different times, as a function of the initial value, for the $\mathrm{L}$ interaction (left part). $n=2,3,5$ are considered. Distribution of $\sigma_{k}$, for the same $n$ numbers, as a function of time for the ensemble of randomly initialized events (right part).

Same as Fig.10, for $n=5,6,7$.

Figure 11:

Figure 12:

Same as Fig.10, but for the $\mathrm{S}$ interaction and $n=5,10,15$.

Figure 13:

Same as Fig.10, but for the $\mathrm{S}$ interaction and $n=15,20,25$.

Figure 14:

Lyapunov exponent (squares), as defined in ref.[7], as a function of $T$ (upper part), and $\rho$ (lower part), compared with the largest imaginary frequency of the linear response, as predicted from the analytical dispersion relation (see ref.[2]) (full line), in the case of the $\mathrm{S}$ interaction.

Figure 15:

Lyapunov exponent as a function of time obtained from randomly initialized events, with average density $\rho=0.4 \rho_{0}$.

Solid line: the exact simulation; dashed line: the linearized simulation; dashdotted line: the simulation obtained using decoupled non-linear oscillators.

The value of the frequency of the most unstable mode, $\omega_{k_{\max }}$, is also reported, as the dotted straight line.

Figure 16:

Fragment size distribution computed for different values of the cut parameter: $\rho_{\text {cut }}=0.275 \mathrm{fm}^{-2}$ (thin solid line), $\rho_{\text {cut }}=0.1 \mathrm{fm}^{-2}$ (dashed line), $\rho_{c u t}=0.05 \mathrm{fm}^{-2}$ (thick solid line). Two different times are considered. 

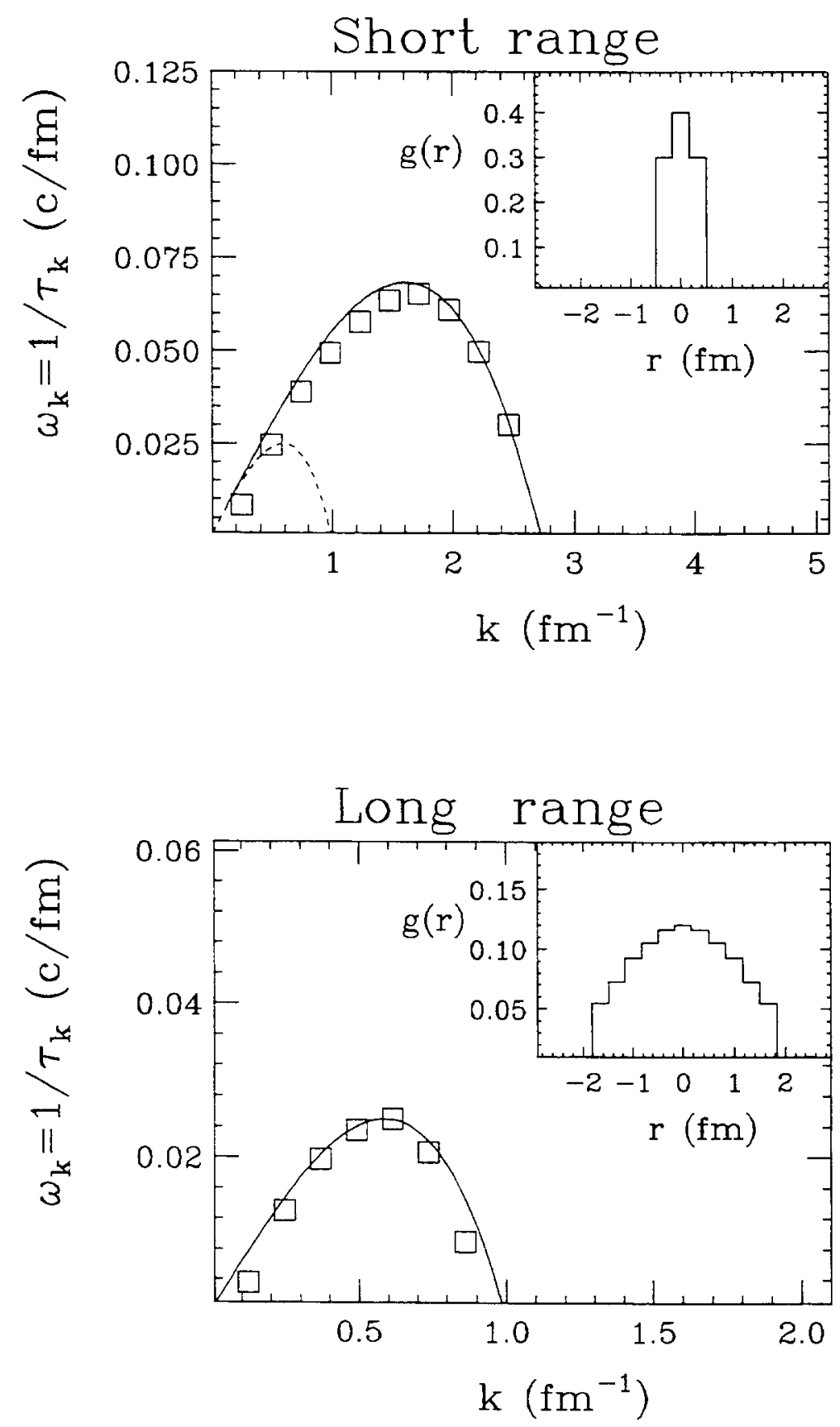

Fig 1 

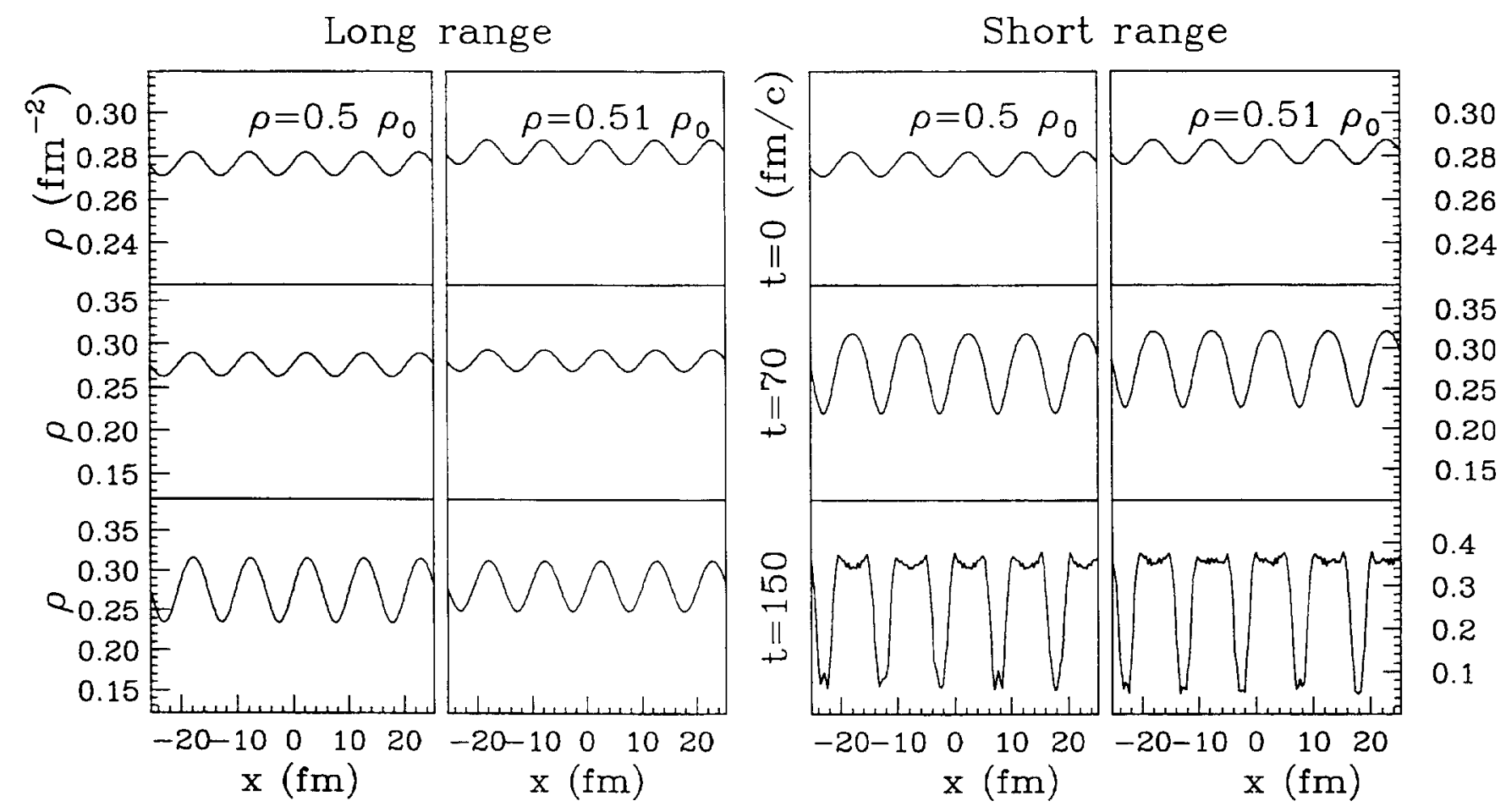

F.g. 2 

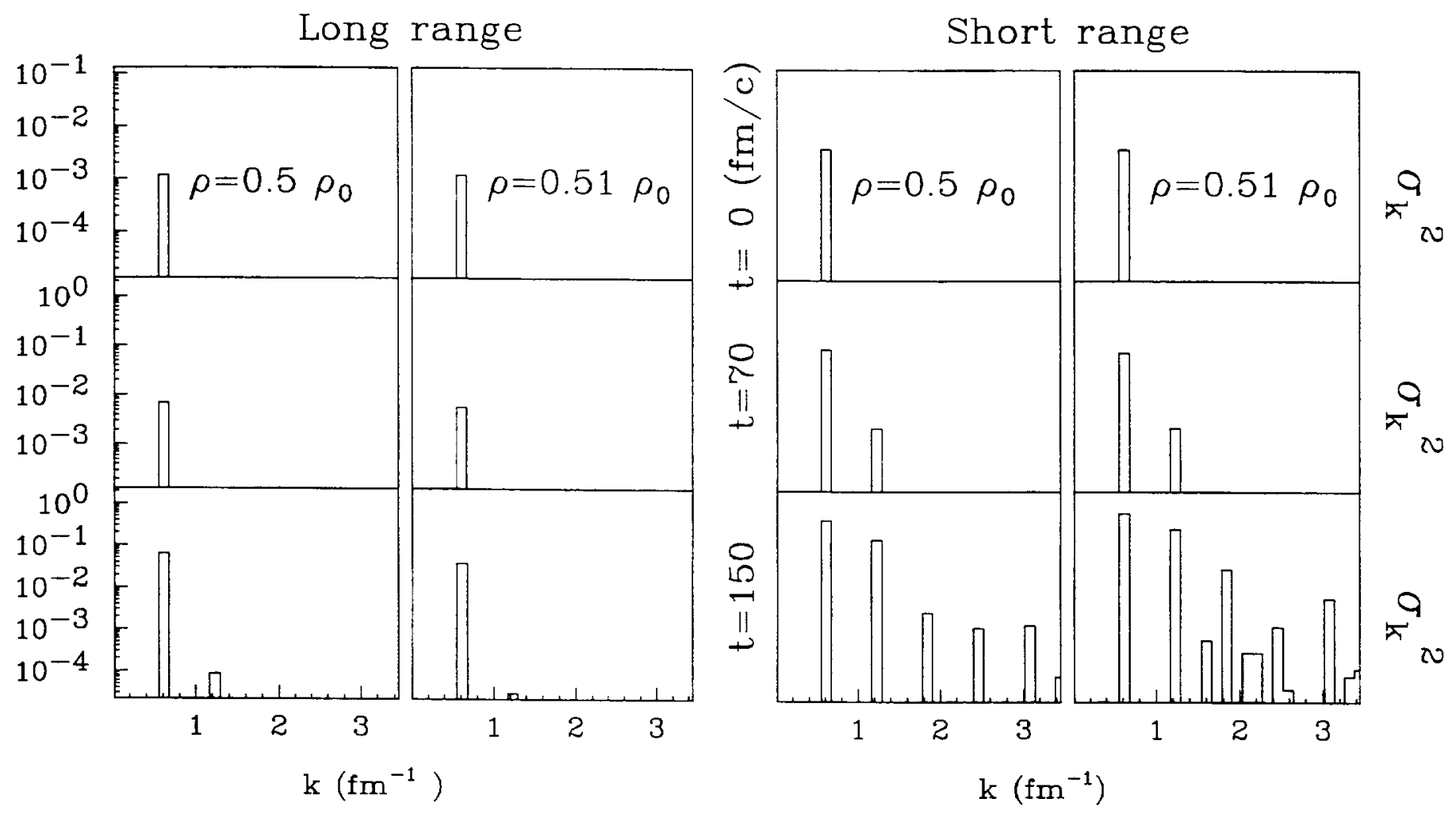

Fig. 3 
(a) long range

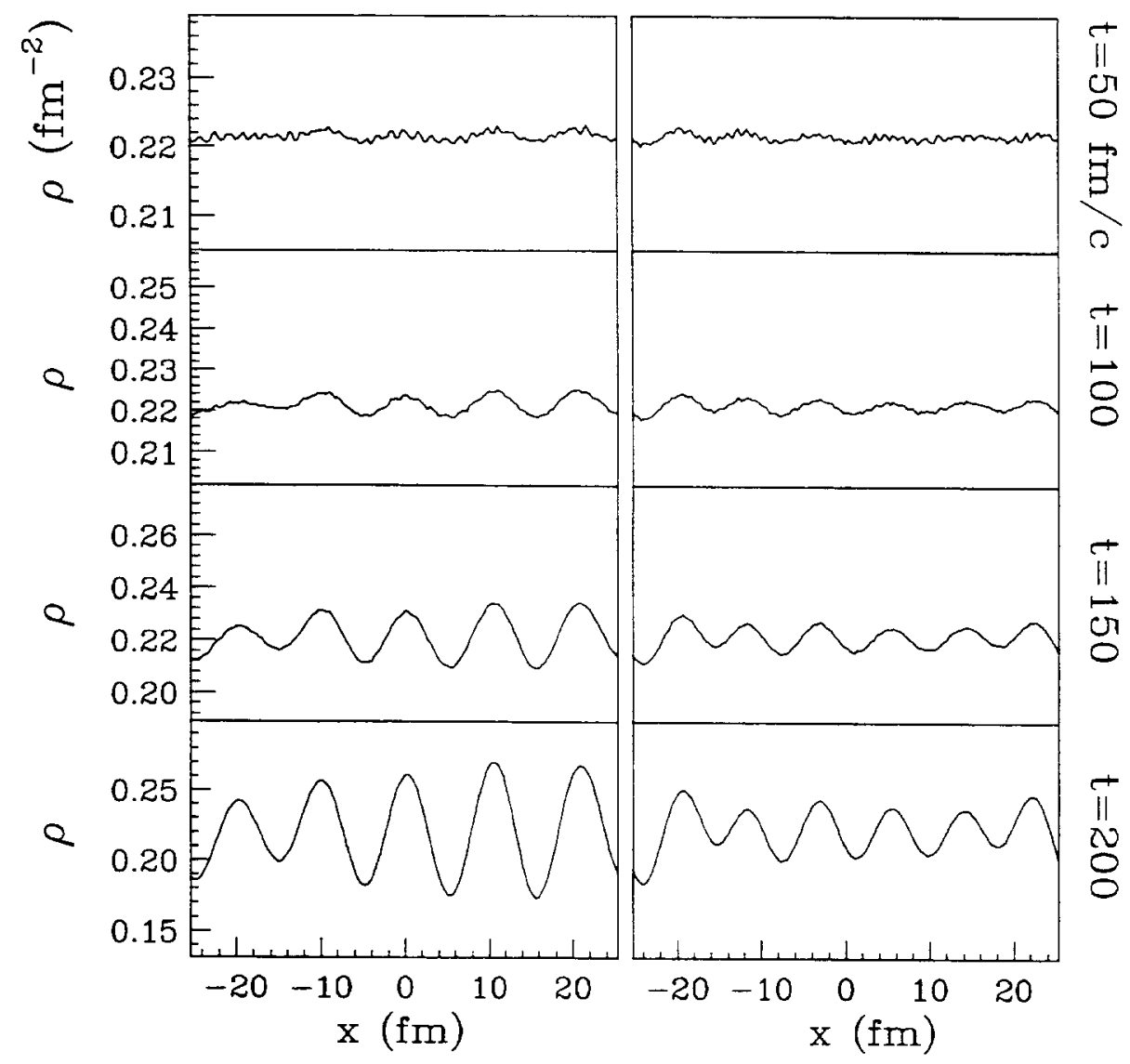

Fig.4a) 
(b) short range

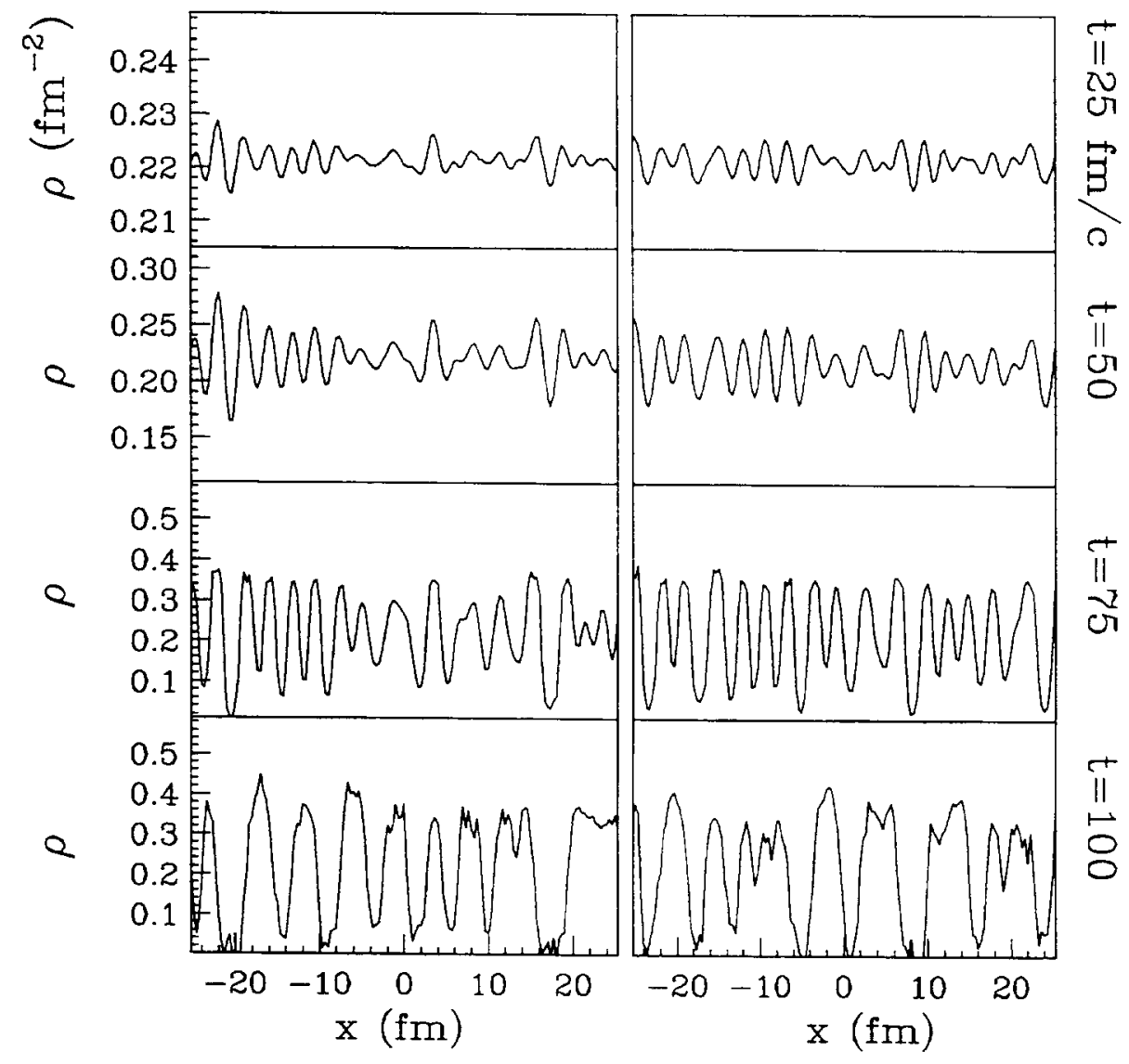

Fig.t b) 


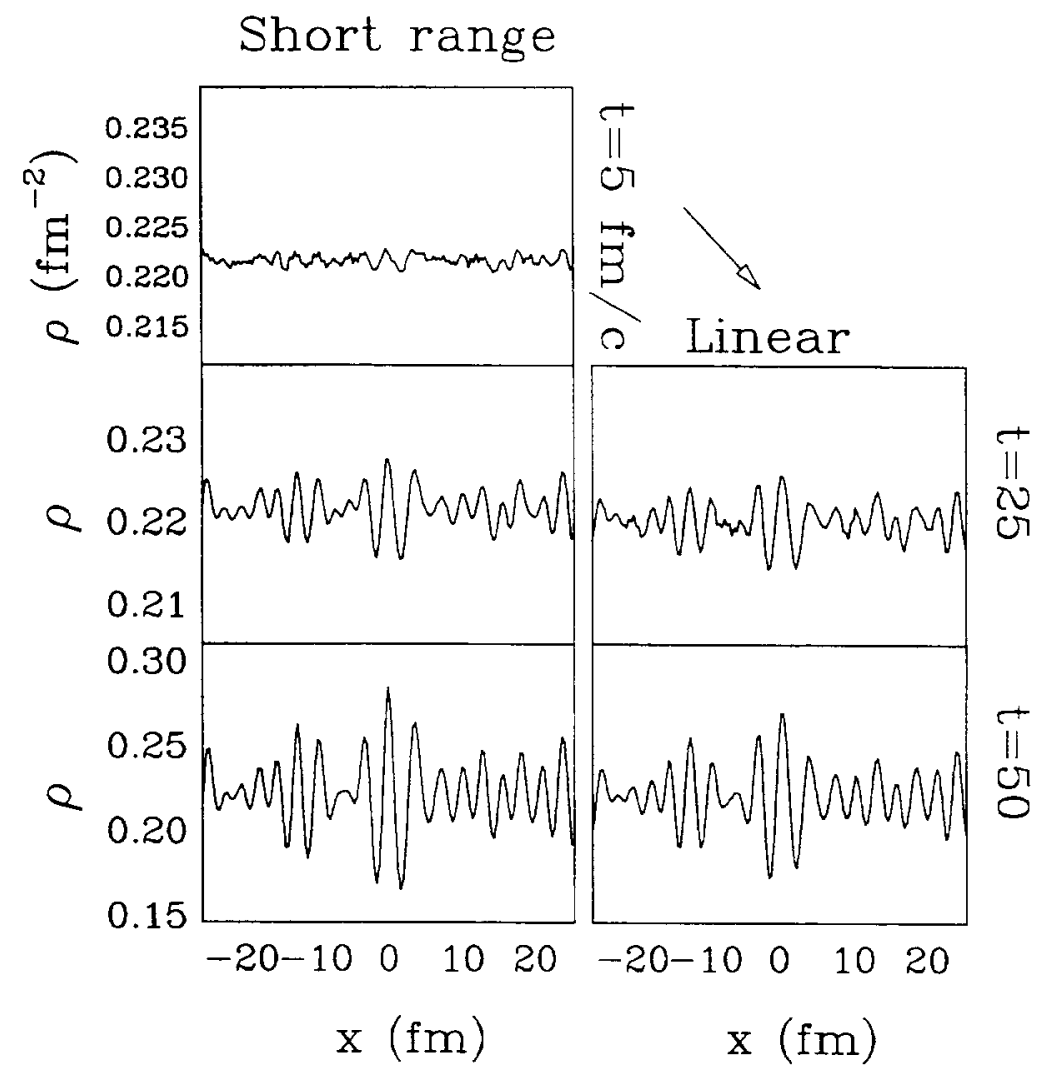

Fig. 5 

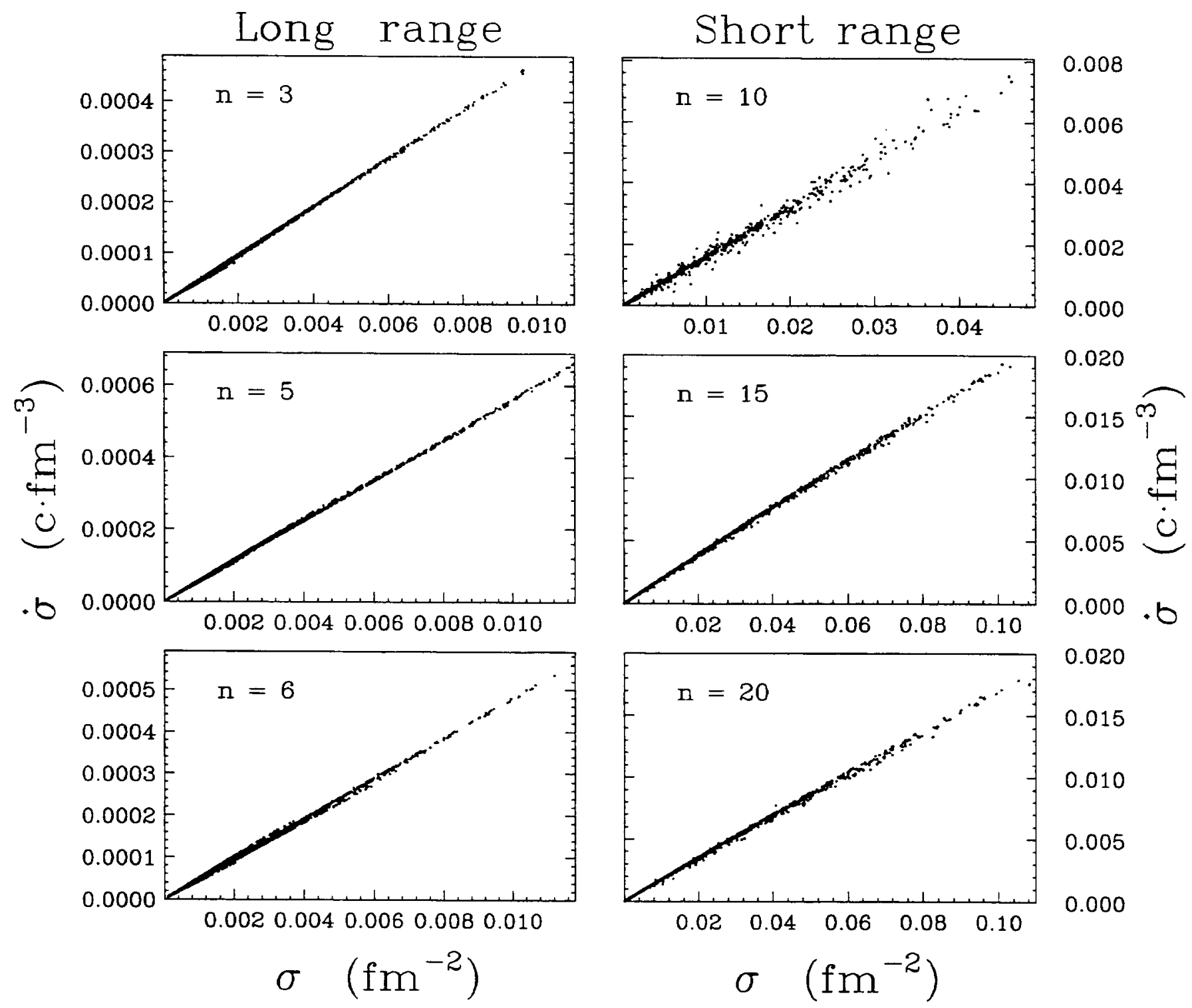

Fif. 6 


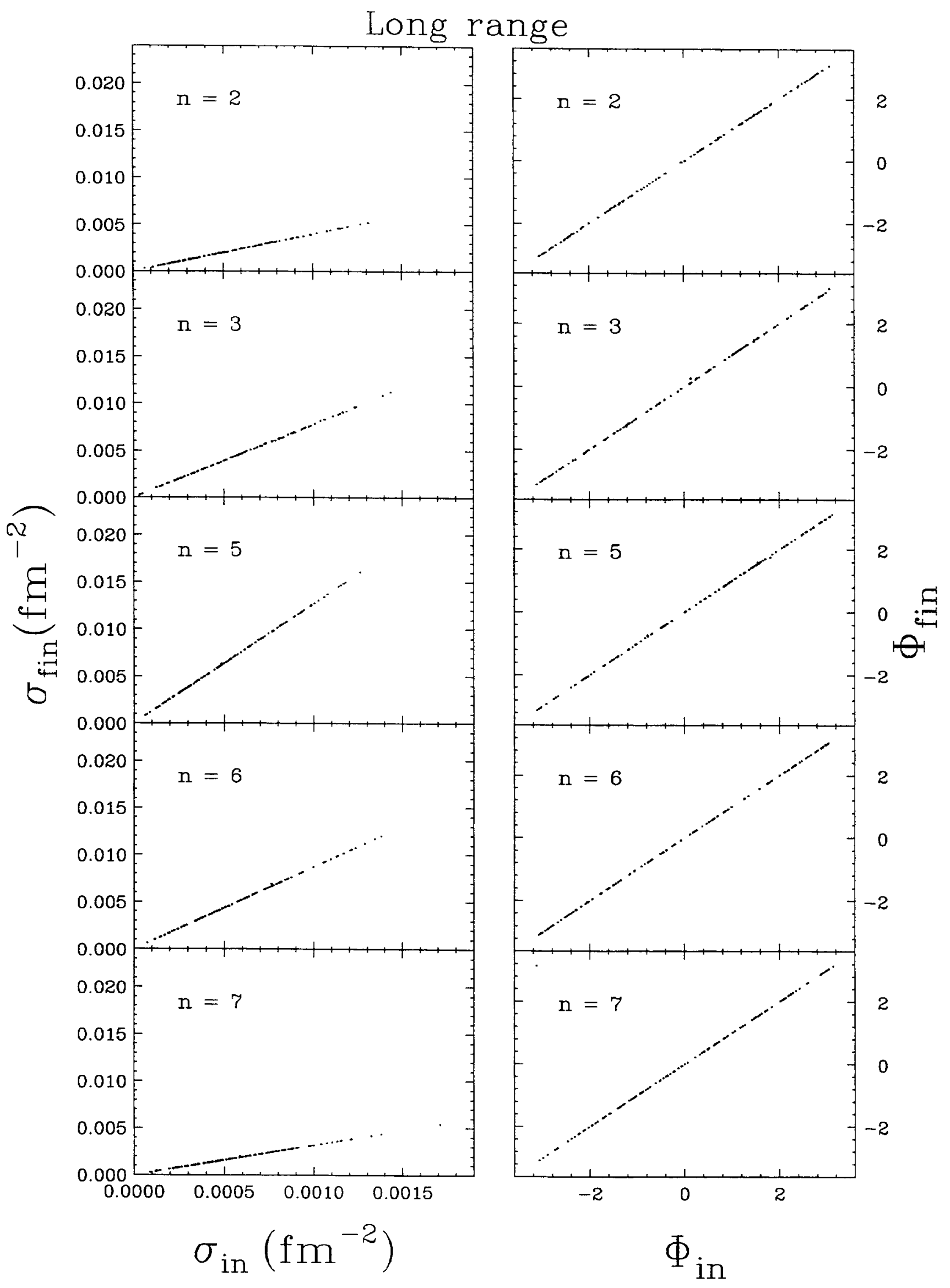

Fif. 7 
Short range
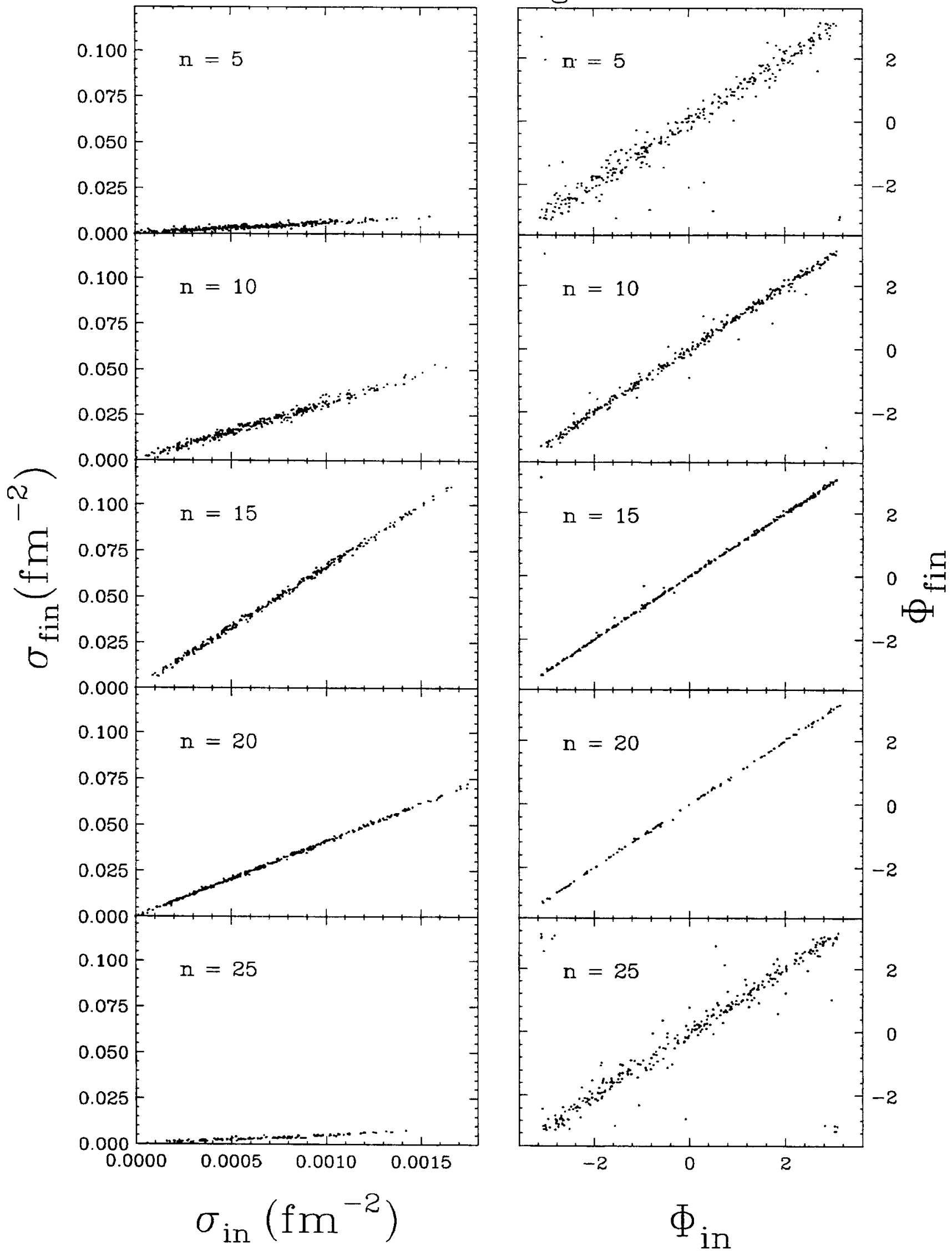

Fif. 8 
(a) Long range; $n=5$

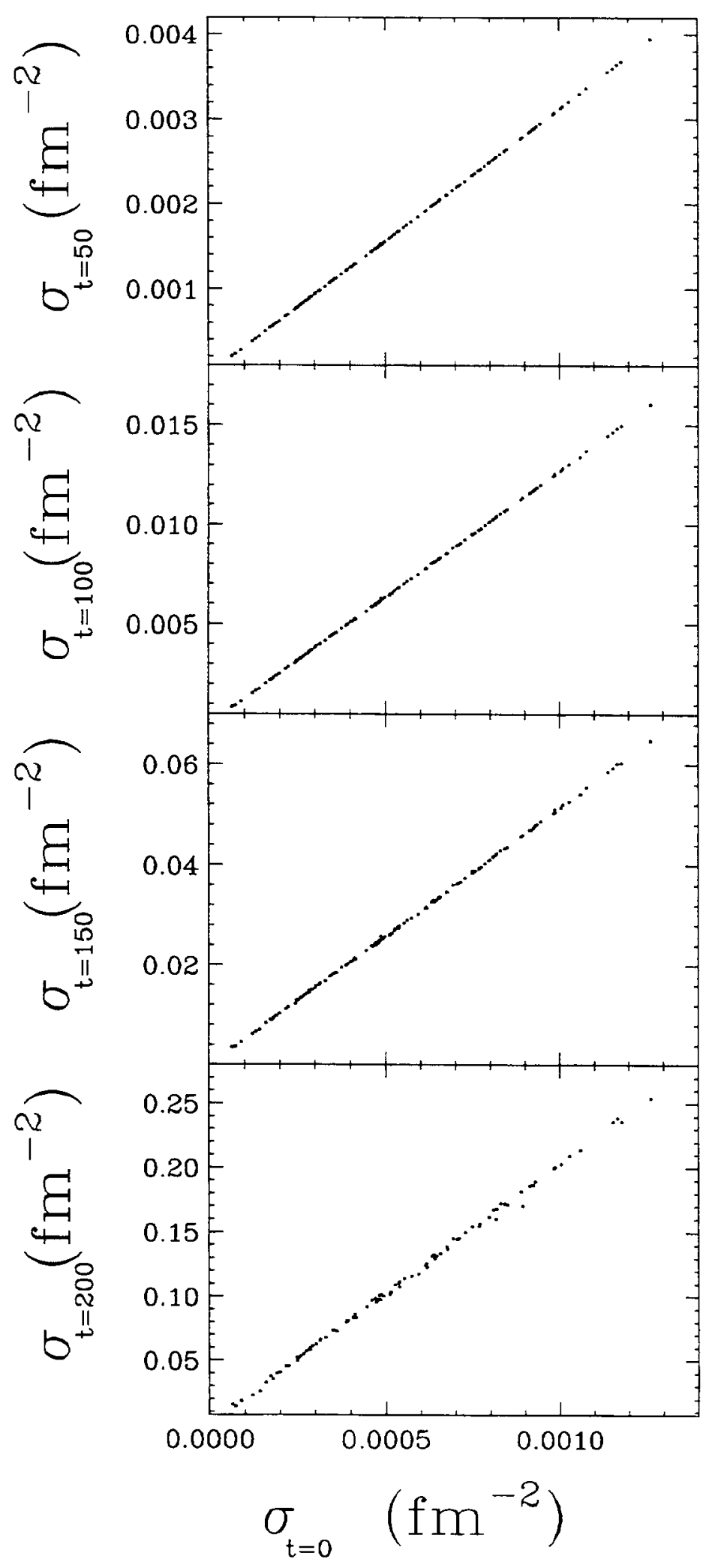

Fig. $\rho$ a) 
(b) Short range; $n=14$

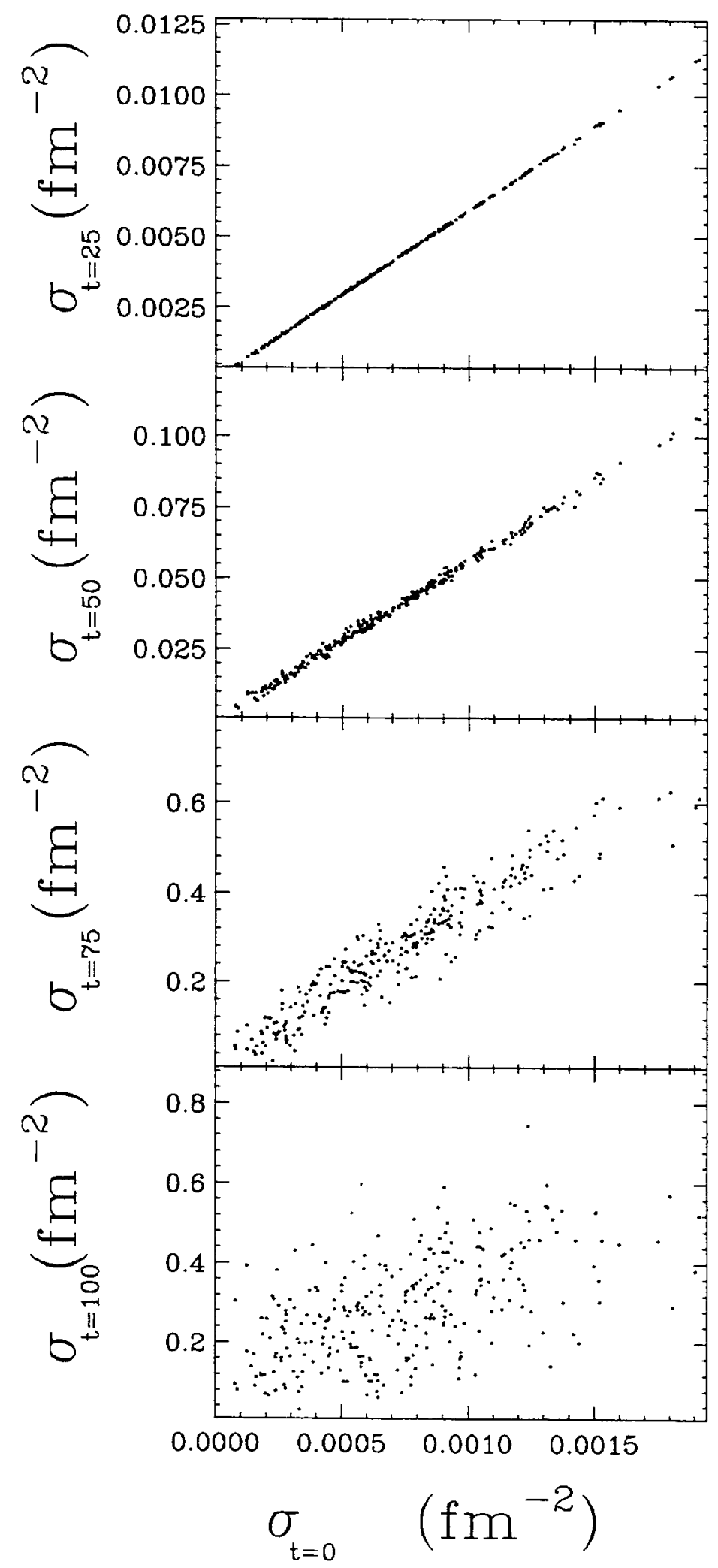

Fig. 9 b) 
Long range

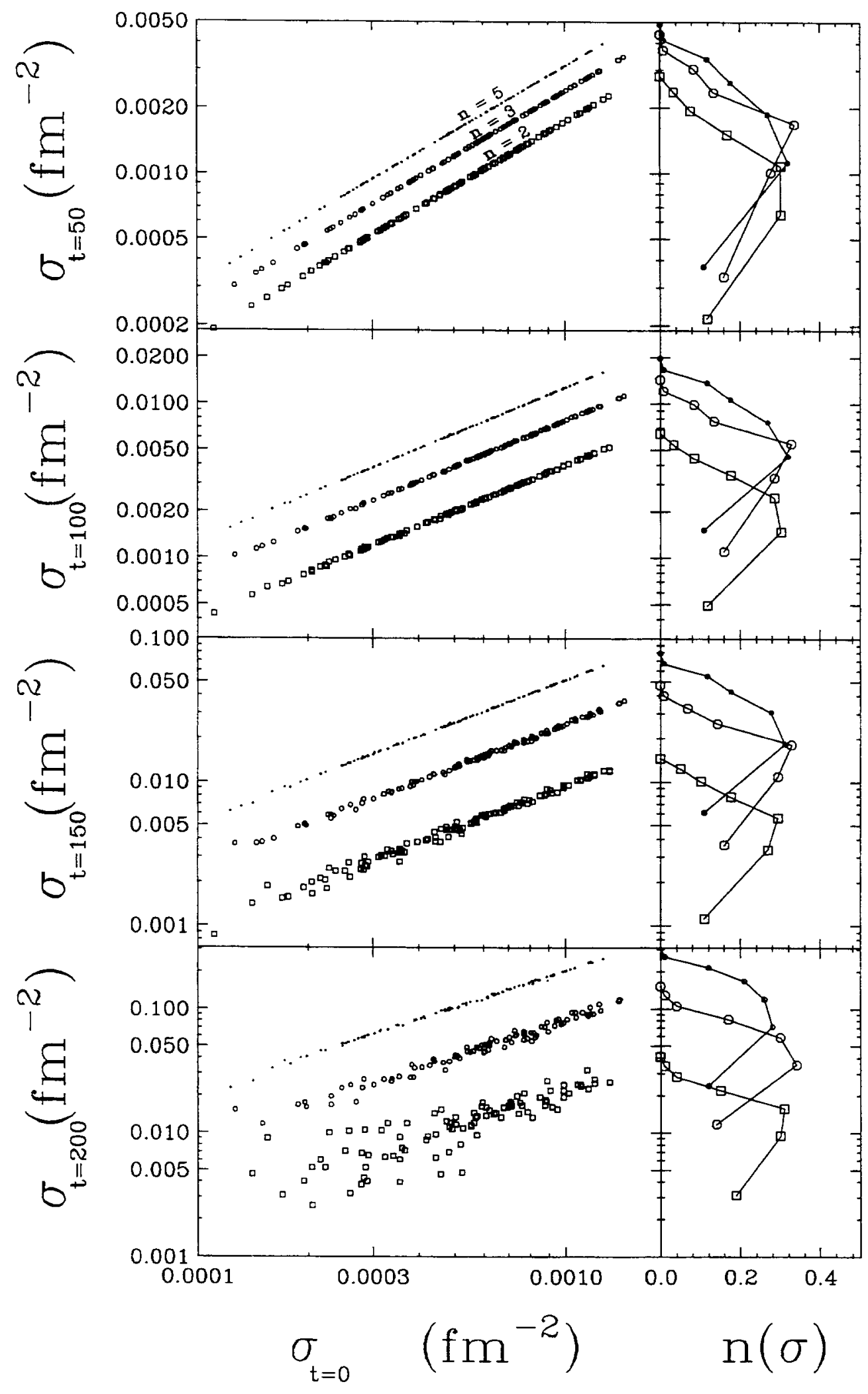

Fig. 10 


\section{Long range}

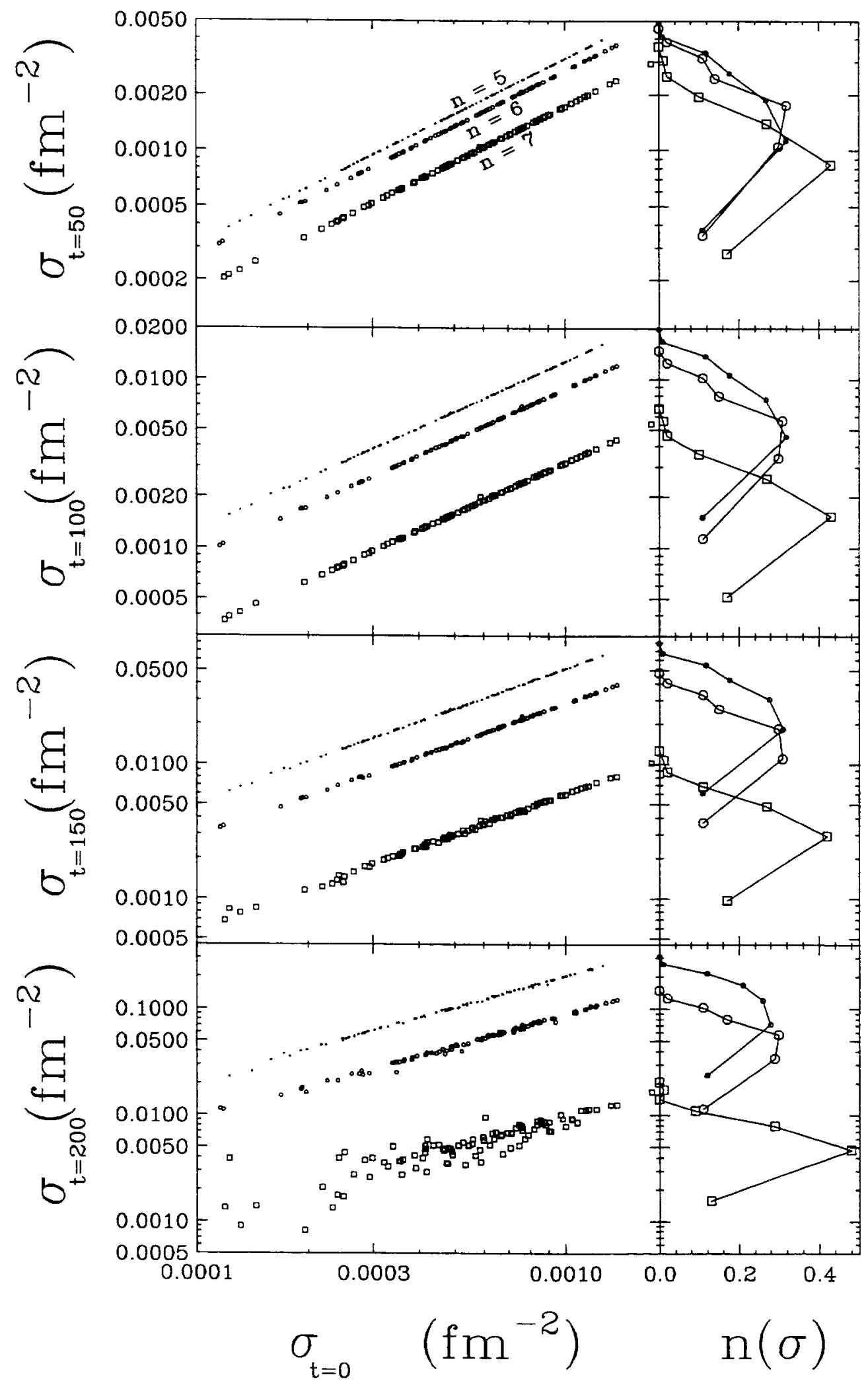

Fig. 11 
Short range

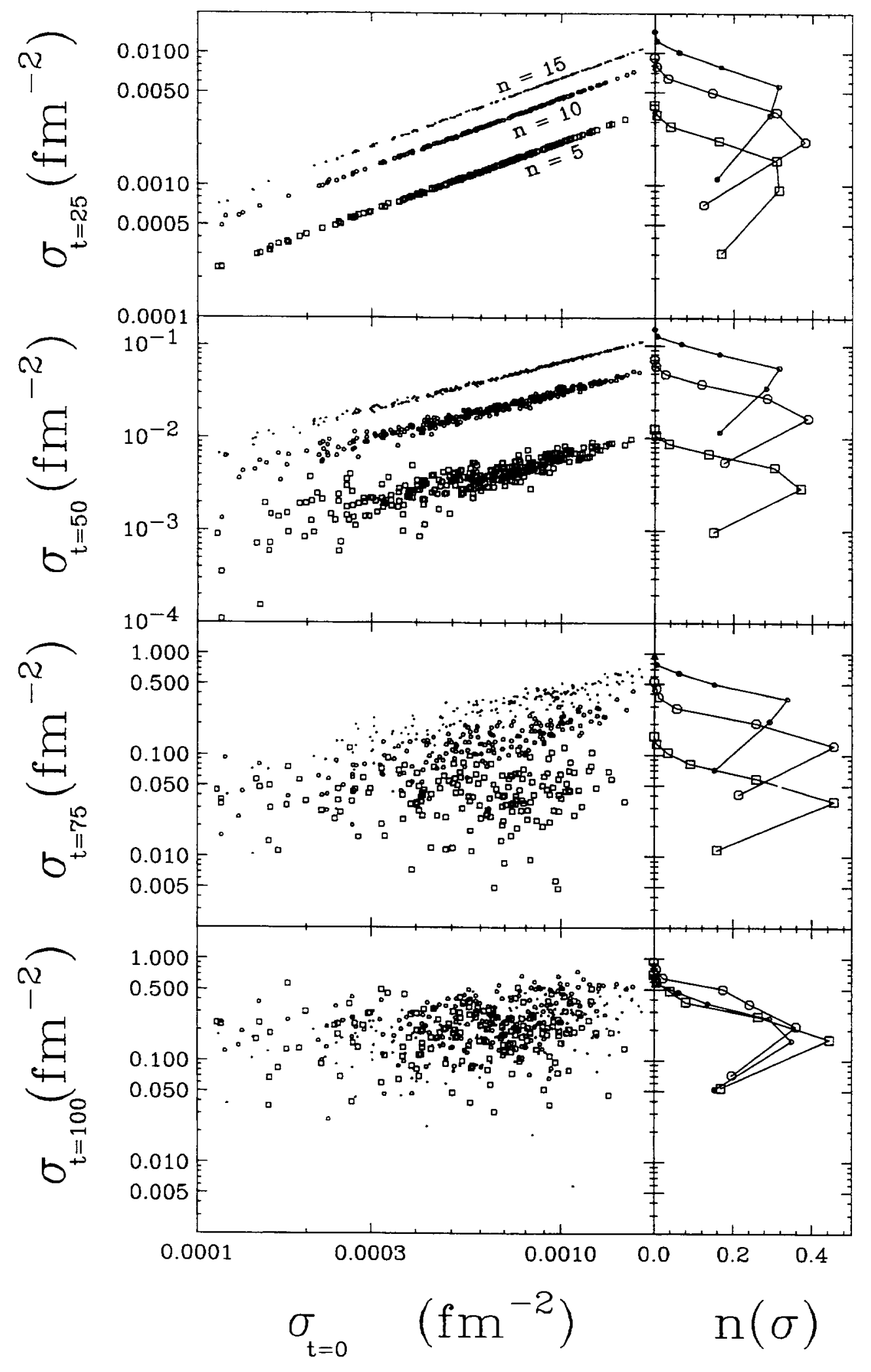

Fig. 12 
Short range

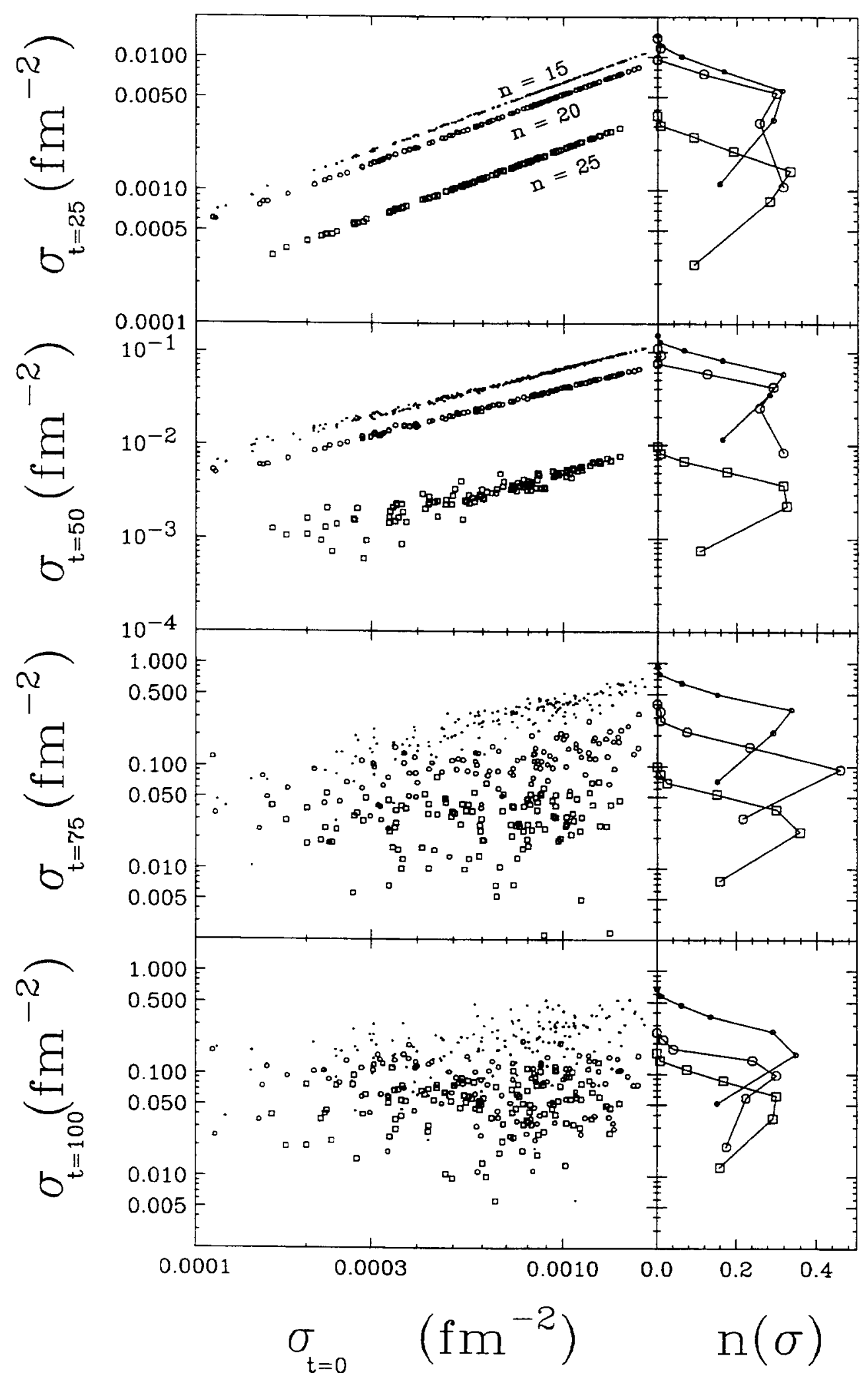



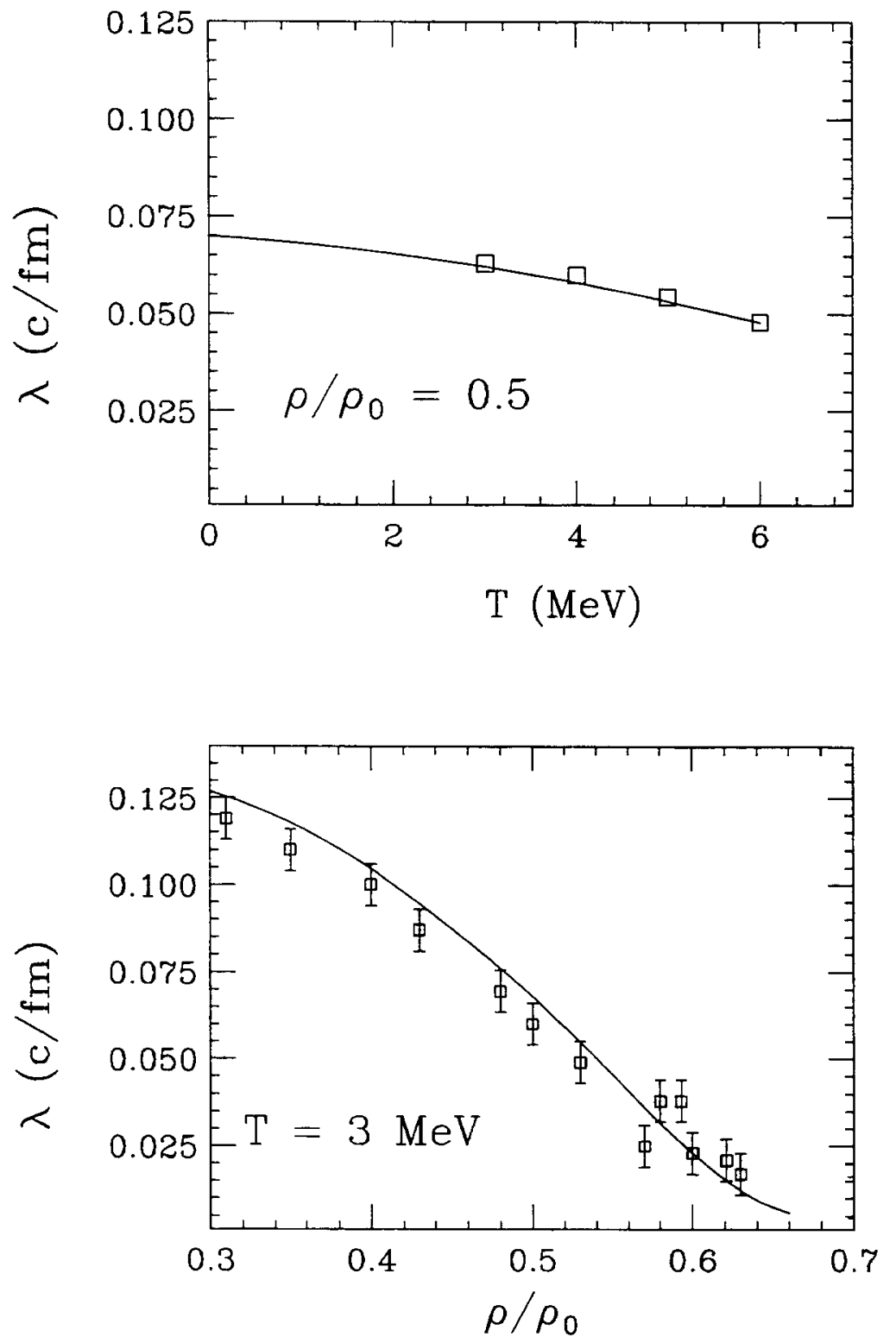

Fig. 14 


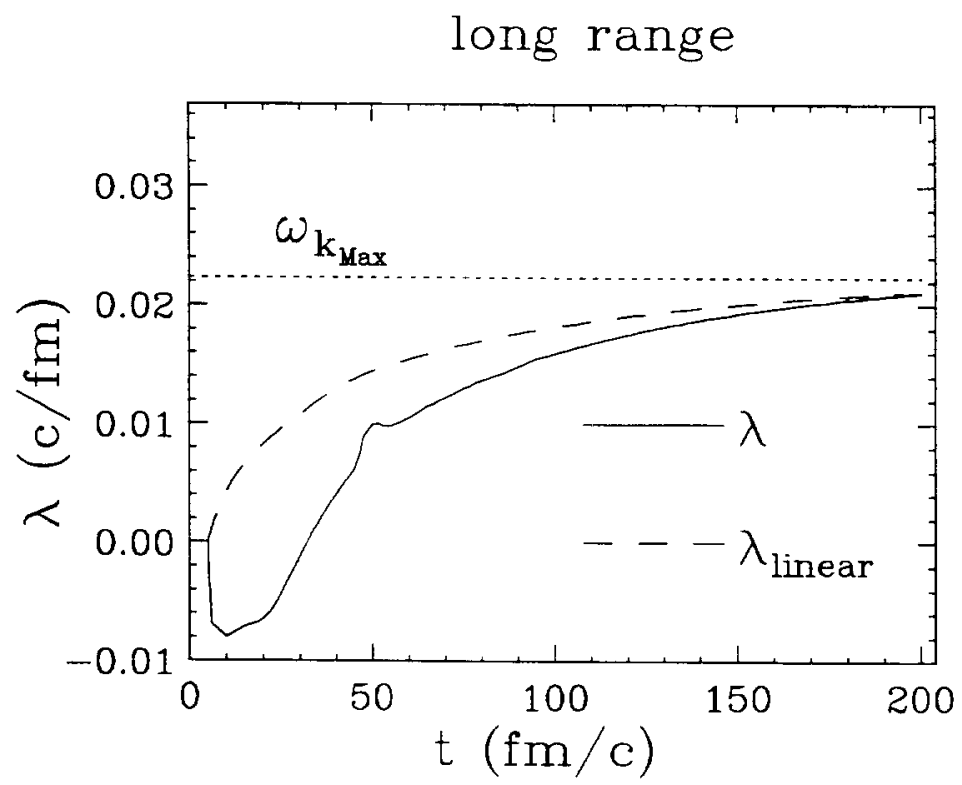

short range

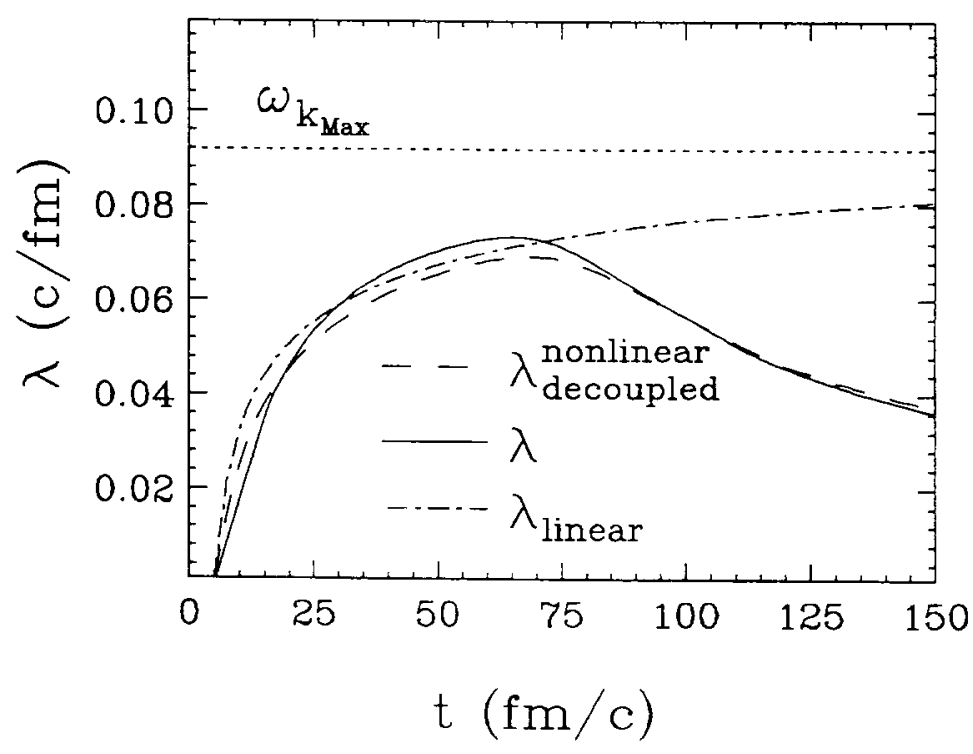

Fig. 15 

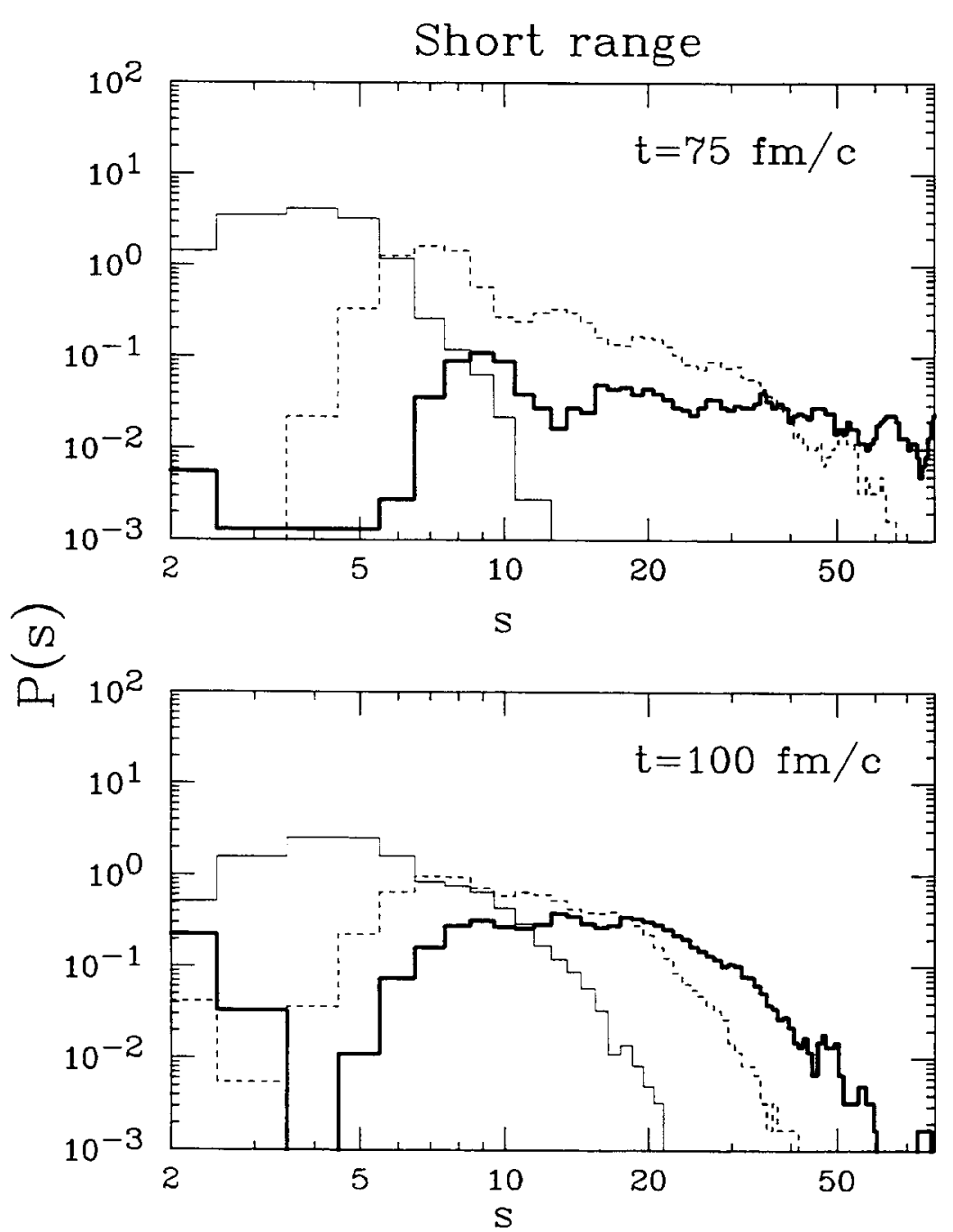

FRAGMENT SIZE DISTRIBUTION 


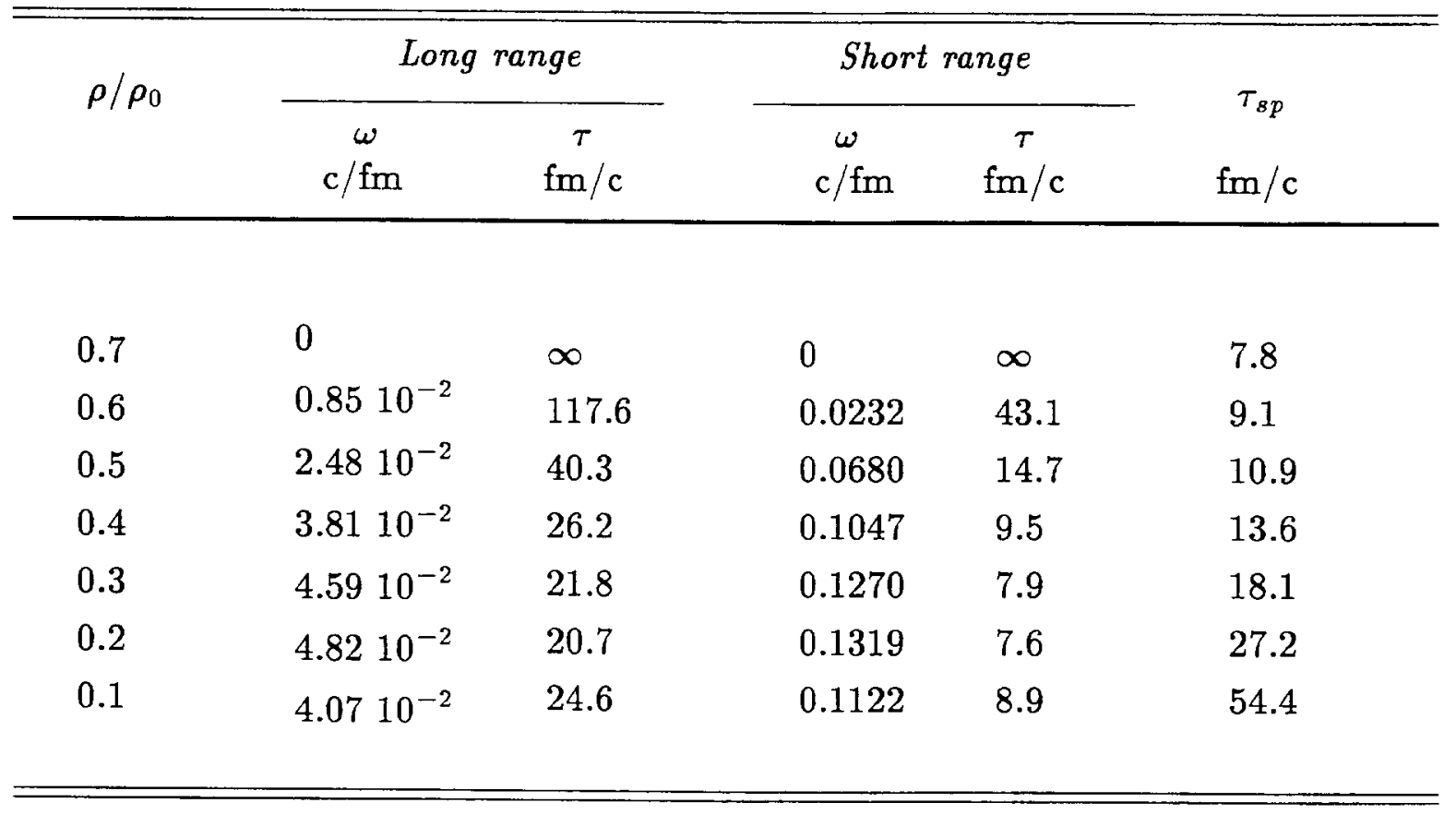

Table I 